\title{
Modeling Unentangled Polystyrene Melts in Fast Elongational Flows
}

\author{
Giovanni Ianniruberto, ${ }^{*}()^{\circ}$ Antonio Brasiello, ${ }^{(0)}$ and Giuseppe Marrucci \\ Department of Chemical, Materials, and Production Engineering, Federico II University, Piazzale Tecchio 80, 80125 Napoli, Italy
}

\author{
Supporting Information
}

ABSTRACT: Recent data of unentangled polystyrene melts in startup elongational flow and subsequent relaxation [Matsumiya, Y.; Watanabe, H.; Masubuchi, Y.; Huang, Q.; Hassager, O. Nonlinear Elongational Rheology of Unentangled Polystyrene and Poly(p-Tert-Butylstyrene) Melts. Macromolecules 2018, 51 (23), 9710-9729] are here compared to the predictions of Brownian simulations of Fraenkel chains endowed with a monomeric friction coefficient that decreases with increasing the order parameter of the Kuhn segments. The model compares favorably with all startup data if a time delay in reducing the

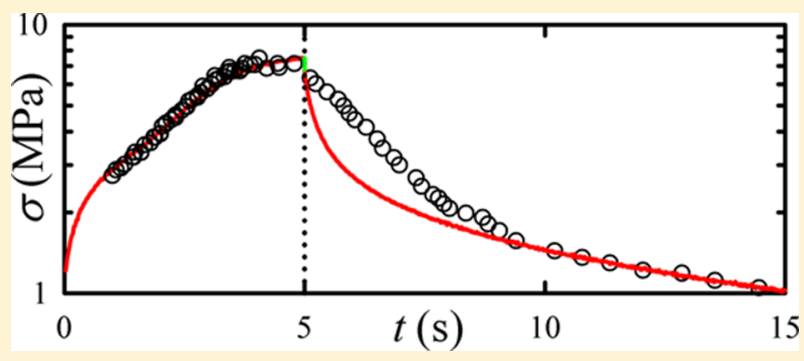
monomeric friction coefficient is accounted for in the fastest flows, a feature already observed by Matsumiya et al. The same model also describes the relaxation data except for a minor discrepancy in the longest relaxation time, probably due to the imperfect monodispersity of the sample and/or to a minor influence of topological interactions, not strong enough to be called entanglements. A discrepancy is also observed at the beginning of relaxation after fast flows, the origin of which remains unexplained.

\section{INTRODUCTION}

Molecular modeling of the rheology of entangled polymers has been developed over many years starting from the fundamental contributions of de Gennes ${ }^{1}$ and of Doi and Edwards. ${ }^{2}$ However, only in recent times, it was found necessary to account for an effect that is important in fast flows: the friction coefficient $\zeta$ of the monomeric unit (Kuhn segment) changes because of the flow-induced co-alignment of the molecules. ${ }^{3}$ Specifically, for polystyrene (PS), $\zeta$ is found to decrease in fast elongational flows, seemingly even by a large amount. The $\zeta$ reduction has been linked to the increase in the order parameter of the Kuhn segments, although the relationship between them (expected to be chemistry-dependent) cannot easily be predicted theoretically. However, such relationship has been determined through molecular dynamics of shear flows of PS oligomers, ${ }^{4}$ as well as from stress relaxation data following elongational flows of high-molar-mass PS melts. ${ }^{5}$

Very recently, $\zeta$ reduction has been unequivocally demonstrated by the elongational flow data of unentangled PS melts reported by Matsumiya et al. ${ }^{6}$ As emphasized by those authors, the advantage of using unentangled polymers instead of entangled ones resides in the fact that, for the latter, other nonlinear effects are simultaneously present (convective constraint release, disentanglement), which are absent in unentangled polymers. Moreover, in unentangled polymers, orientation and stretch take place simultaneously at the onset of the nonlinear response, whereas in entangled ones, nonlinearities start because of flow-induced "tube" orientation while chain stretch (which reduces $\zeta$ ) occurs only in faster flows (faster than the reciprocal Rouse time of the chain). ${ }^{2} \mathrm{~A} \zeta$ reduction effect in unentangled polymers had also been noted by Masubuchi et al. ${ }^{7}$ through Kremer-Grest (bead-spring chains) molecular dynamics simulations of stress relaxation after cessation of fast elongational flows. However, the Kremer-Grest "chemistry" gave rise to a $\zeta$ reduction effect much smaller than that found by Matsumiya et al. ${ }^{6}$ The dependence of the $\zeta$ reduction effect on the chemistry of the polymer chain has been confirmed by Matsumiya et al., ${ }^{6}$ who, together with PS, also report experiments on poly(p-tertbutylstyrene) unentangled melts.

In analyzing their data, Matsumiya et al. ${ }^{6}$ model the unentangled polymer as a finitely extensible (FENE) beadspring Rouse chain with a pre-averaged FENE effect. The $\zeta$ reduction is first estimated by them from the elongational steady-state results in the form of a dependence of $\zeta$ on the elongational stress. However, they find that the same dependence cannot explain the transient startup data, and even less the relaxation data following cessation of elongational flow. They argue that the model failure in the transient results might be due to a delay in the $\zeta$ reduction with respect to the flow-induced stretch/orientation of the polymer chains.

In the present paper, we analyze the PS27 data of Matsumiya et al. ${ }^{6}$ (PS melt with molar mass $27.1 \mathrm{k}$ ) using a more realistic model of the unentangled polymer, namely, a chain with the appropriate number of inextensible Kuhn segments. The quasiinextensibility of a Kuhn segment is modeled using stiff Fraenkel springs. ${ }^{8}$ Hence, the Fraenkel chain used here automatically accounts for the finite extensibility of the polymer, without introducing a maximum extensibility parameter. Furthermore, no pre-averaging is needed, as we

Received: April 1, 2019

Revised: May 22, 2019

Published: June 5, 2019 
perform Brownian dynamics simulations of a large number of molecules, and the $\zeta$ reduction effect is directly linked to the order parameter of the Kuhn segments of all molecules of the simulation.

The paper is organized as follows. We describe the model in Section 2. Next, in Section 3, we compare predictions to the PS27 data of Matsumiya et al. ${ }^{6}$ and discuss results. Final remarks in Section 4 conclude the paper.

\section{MODEL}

In unentangled polymer melts, each chain feels the surrounding chains through the excluded volume interaction (preserving the mass density and presumably not affecting chain dynamics) and through the monomeric friction coefficient $\zeta^{2}$. Then, assuming that the Kuhn segments are essentially inextensible, unentangled polymers can be modeled as Fraenkel chains made up of beads endowed with the friction coefficient $\zeta$ and connectors of root-mean-square Kuhn length $l_{\mathrm{K}}$ having a suitably large spring constant $H$. Previously, the Fraenkel chain model has been successfully used in describing the rheology of dilute polymer solutions, ${ }^{9}$ and its use for unentangled melts appears sensible.

The Fraenkel spring equation reads

$$
\mathbf{F}=H\left(l-l_{\mathrm{K}}\right) \mathbf{u}
$$

where $\mathbf{F}$ is the force in the chain segment having orientation described by the unit vector $\mathbf{u}$ and $H$ is so chosen that the current segment length $l$ remains close to $l_{\mathrm{K}}$.

Chain dynamics is described by the following Langevin equation

$$
\begin{gathered}
\zeta\left(\frac{\mathrm{d} \mathbf{R}_{i}}{\mathrm{~d} t}-\mathbf{k} \cdot \mathbf{R}_{i}\right)=\mathbf{F}_{i+1}-\mathbf{F}_{i}+\mathbf{f}_{i} \quad i=0, \ldots, N, \\
\mathbf{F}_{0}=\mathbf{F}_{N+1}=\mathbf{0}
\end{gathered}
$$

where $\mathbf{R}_{i}$ is the position of the $i$ th bead, $\mathbf{k}$ is the velocity gradient, and $\mathbf{F}_{i}$ obeys eq 1 (for $1 \leq i \leq N$ ) with $l_{i}$ and $\mathbf{u}_{i}$ obeying the connector vector relationship $\mathbf{Q}_{i} \equiv \mathbf{R}_{i}-\mathbf{R}_{i-1}=l_{i} \mathbf{u}_{i}$. The Brownian force $\mathbf{f}_{i}$ has a Gaussian distribution with $\left\langle\mathbf{f}_{i}\right\rangle=\mathbf{0}$, and $\left\langle\mathbf{f}_{i}(t) \mathbf{f}_{i}\left(t^{\prime}\right)\right\rangle=2 \zeta k_{\mathrm{B}} T \delta\left(t-t^{\prime}\right) \mathbf{I}$, where $\langle\ldots\rangle$ indicates ensemble average, $k_{\mathrm{B}} T$ is the thermal energy, $\delta(\cdot)$ is the Dirac delta function, and $\mathbf{I}$ is the unit tensor.

Observables are computed by averaging over a suitably large population of chains. The observables of interest are the stress tensor $\boldsymbol{\sigma}$ and the Kuhn segment order parameter tensor $\mathbf{S}$, respectively, given by

$$
\begin{aligned}
& \boldsymbol{\sigma}=\nu\left\langle\sum_{i=1}^{N} \mathbf{F}_{i} \mathbf{Q}_{i}\right\rangle \\
& \mathbf{S}=\frac{1}{N} \sum_{i=1}^{N} \mathbf{S}_{i}, \quad \mathbf{S}_{i}=\frac{3}{2}\left(\left\langle\mathbf{u}_{i} \mathbf{u}_{i}\right\rangle-\frac{1}{3} \mathbf{I}\right)
\end{aligned}
$$

where $\nu$ is the number density of chains and $S_{i}$ is the "local" order parameter tensor, i.e., that of the Kuhn segments located at the $i$ th position along the chains. In the case of uniaxial elongational flows, the order parameter tensor is fully determined by the scalar order parameter $S$ given by

$$
S=\frac{1}{N} \sum_{i=1}^{N} S_{i}, \quad S_{i}=\left\langle u_{i, x}^{2}-u_{i, y}^{2}\right\rangle
$$

where $u_{i, x}$ and $u_{i, y}$ are components of $\mathbf{u}_{i}$ in a Cartesian coordinate system with the $x$-axis in the elongation direction. Obviously, the order parameter $S$ is zero at equilibrium, while approaching unity when chains become increasingly oriented in the elongation direction.

Consistently with previous results, ${ }^{4,5,10,11}$ it is here assumed that the monomeric friction coefficient $\zeta$ is the following decreasing function of the Kuhn segment order parameter $\boldsymbol{S}$

$$
\frac{\zeta}{\zeta_{\mathrm{eq}}}=\frac{1}{\left[1+\left(\frac{s}{s_{\mathrm{c}}}\right)^{\alpha}\right]^{n / \alpha}}
$$

Since $\alpha$ will be set at a value around 2, eq 6 implies that, for $S$ smaller than a critical value $S_{c}$ (of order 0.1 ), $\zeta$ essentially remains at the equilibrium value $\zeta_{\text {eq }}$, whereas for $S$ larger than $S_{\mathcal{c}} \zeta / \zeta_{\text {eq }}$ follows the power law $\left(S / S_{\mathrm{c}}\right)^{-n}$.

By adopting a procedure similar to that used by Matsumiya et al., ${ }^{6}$ the parameters $S_{\mathcal{O}} \alpha$, and $n$ are determined by fitting predictions to the steady-state values of the elongational viscosity (see Figure 4 below). A comparison of our friction reduction law (eq 6) to that used by Matsumiya et al. ${ }^{6}$ is reported in the Supporting Information. As noted by Matsumiya et al., ${ }^{6}$ we will also observe (in Figure 2 below) that eq 6 does not fit some of the transient results of elongational startup, suggesting that a time delay be introduced. We here propose that, during a transient, the effective monomeric friction coefficient $\zeta_{\text {eff }}$ to be used in the Brownian dynamics in place of $\zeta$ is linked to the function $\zeta(S)$ given by eq 6 through a time-differential equation of a simple form

$$
\frac{\mathrm{d} \zeta_{\mathrm{eff}}}{\mathrm{d} t}=-\frac{1}{\tau}\left(\zeta_{\mathrm{eff}}-\zeta\right)
$$

Note however that in eq 7 both $\zeta$ and $\tau$ are time-dependent, $\zeta$ because of the evolution of the order parameter $S$ while $\tau$ because all relaxation times are assumed to be proportional to $\zeta$ through equations of the form

$$
\frac{\tau}{\tau_{\mathrm{eq}}}=\frac{\zeta}{\zeta_{\mathrm{eq}}}
$$

where $\tau_{\mathrm{eq}}$ is the value of $\tau$ at equilibrium.

Simulations were actually run in nondimensional units, using $l_{\mathrm{K}}, k_{\mathrm{B}} T$, and $\zeta_{\text {eq }} l_{\mathrm{K}}^{2} / k_{\mathrm{B}} T$ as units of length, energy, and time, respectively. The unit of time also represents the relaxation time $\tau_{\mathrm{K}}$ of the Kuhn segment at equilibrium. In comparing simulation results to the PS27 data, known values of $l_{\mathrm{K}}$ and $k_{\mathrm{B}} T$ are used, while $\tau_{\mathrm{K}}$ is determined by fitting linear viscoelastic data.

Before comparing predictions to the PS27 data, however, it is worth recalling that, since unentangled melts have a relatively fast relaxation, the glassy dynamics provides a minor but non-negligible contribution to the rheological response. ${ }^{6}$ For the glassy contribution, we will use the experimental results of Inoue et al. $^{12}$ for the PS chemistry.

\section{RESULTS AND DISCUSSION}

3.1. Linear Viscoelasticity. For the linear rheology of unentangled melts, it is not necessary to use the model described in the previous section. Indeed, the Rouse model is expected to be adequate, provided the glassy dynamics is also accounted for. ${ }^{13}$ The parameters of the Rouse model are the 
modulus $G=v k_{\mathrm{B}} T$ and the Rouse time $\tau_{\mathrm{R}}$. The chain number density is given by $\nu=\rho \mathcal{N} / M$, with $\rho$ the polymer melt density at temperature $T, \mathcal{N}$ the Avogadro number, and $M$ the polymer molar mass. The Rouse time is given by $\tau_{\mathrm{R}}=\frac{6}{\pi^{2}} \frac{\eta_{0}}{G}$, where the zero-shear viscosity $\eta_{0}$ is given by $G^{\prime \prime} / \omega$ in the terminal region, after subtracting the glassy contribution.

The storage and loss glassy moduli of PS27 at $115^{\circ} \mathrm{C}$ were obtained from the data of Inoue et al. ${ }^{12}$ at $116^{\circ} \mathrm{C}$, shifted to $115{ }^{\circ} \mathrm{C}$ using the shift factor reported by Inoue et al. themselves, and are shown in Figure 1 (red curves). The glassy

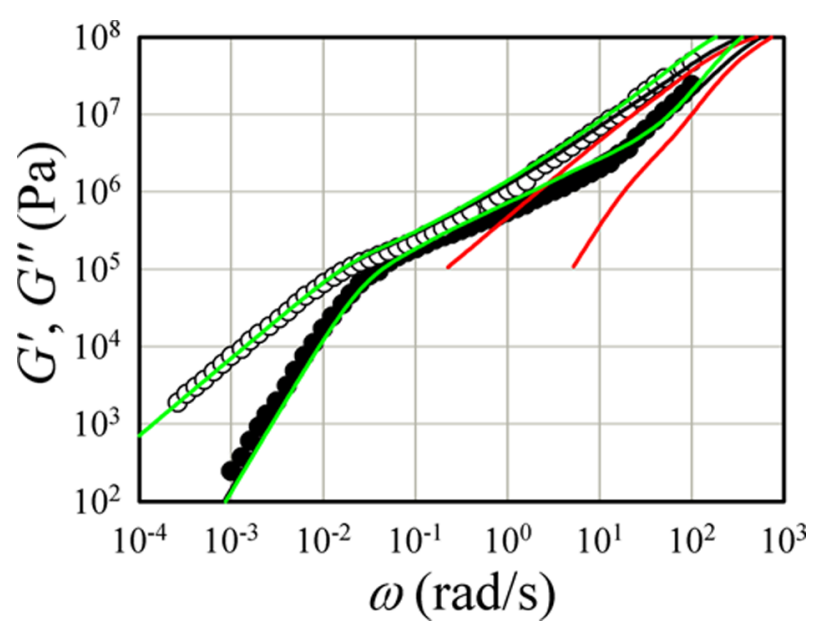

Figure 1. Frequency response of PS27 at $115^{\circ} \mathrm{C}$. Symbols are $G^{\prime}, G^{\prime \prime}$ data from Matsumiya et al. ${ }^{6}$ Red curves are the glassy moduli from Inoue et al. ${ }^{12}$ Black and green curves are Rouse and Fraenkel-chain predictions, respectively, inclusive of the glassy contribution.

contribution to the zero-shear viscosity is therefore estimated (from $G^{\prime \prime}$ glassy) to be $0.5 \mathrm{MPa}$ s. Figure 1 also reports the $G^{\prime}$, $G^{\prime \prime}$ data of PS27 at $115{ }^{\circ} \mathrm{C}$ taken from Matsumiya et al., ${ }^{6}$ revealing a zero-shear viscosity of 7.1 MPs s. Hence, it is $\eta_{0}=$ $7.1-0.5=6.6 \mathrm{MPa}$ s. Since $G=0.12 \mathrm{MPa}$ (the mass density being $\left.\rho=1016 \mathrm{~kg} / \mathrm{m}^{3}\right),{ }^{14}$ the Rouse time is estimated as $\tau_{\mathrm{R}}=$ $33 \mathrm{~s}$. The black curves in Figure 1 (often hidden by the green ones) are obtained by summing the glassy moduli to those of the Rouse model. Good agreement with data (symbols in Figure 1) confirms that PS27 is not entangled.

For the sake of completeness, we have also calculated $G^{\prime}, G^{\prime \prime}$ from the Fraenkel-chain model with the appropriate number, $N$, of Kuhn segments. Since the molar mass of the PS Kuhn segment is $M_{\mathrm{K}}=0.90 \mathrm{k},{ }^{6}$ it is $N=27.1 / 0.9 \cong 30$. As regards the Fraenkel spring stiffness $H$, to save on computational time, we have run most of our simulations with the nondimensional spring constant $\tilde{H}=H l_{\mathrm{K}}^{2} / k_{\mathrm{B}} T$ at the value $\tilde{H}=500$, checking with a few runs with $\tilde{H}=1000$ (as used by Hsieh et al. ${ }^{9}$ for the case of dilute solutions) that the results would not change. As detailed later, higher values of $\tilde{H}$ were also used for very fast flows in the absence of friction reduction. The nondimensional relaxation modulus $G(t)$ was then obtained through the generalized Green-Kubo autocorrelation of several components of the stress tensor at equilibrium ${ }^{15}$

$$
\begin{aligned}
G(t)= & \frac{1}{5} N_{c}\left\{\left[\sigma_{x y}(t) \sigma_{x y}(0)+\sigma_{y z}(t) \sigma_{y z}(0)+\sigma_{z x}(t) \sigma_{z x}(0)\right]\right. \\
& +\frac{1}{6}\left[N_{x y}(t) N_{x y}(0)+N_{y z}(t) N_{y z}(0)+N_{z x}(t)\right. \\
& \left.\left.N_{z x}(0)\right]\right\}
\end{aligned}
$$

where $N_{c}$ (of order $10^{5}$ ) is the number of chains over which the Cartesian stress components $\sigma_{\mathrm{pq}}$ have been calculated from the nondimensional form of eq 3 (with $\nu=1$ ) and $N_{\mathrm{pq}}=\sigma_{\mathrm{pp}}$ $\sigma_{\mathrm{qq}}$. To further improve on statistics, each term in eq 9 [e.g., $\left.\sigma_{x y}(t) \sigma_{x y}(0)\right]$ is replaced by the average over time $\left\langle\sigma_{x y}(t+\right.$ $\left.\left.t^{\prime}\right) \sigma_{x y}\left(t^{\prime}\right)\right\rangle_{t^{\prime}}$. Next, by using open-source software Reptate, ${ }^{16}$ we fitted the $G(t)$ curve with a sum of negative exponentials, hence obtaining the discrete nondimensional spectrum $\left\{G_{i}, \tau_{i}\right\}$ of the Fraenkel chain. To convert the latter to the dimensional spectrum, we multiply the moduli to $v k_{\mathrm{B}} T=0.12 \mathrm{MPa}$ (see above) and the relaxation times to a value of the Kuhn segment relaxation time $\left(\tau_{\mathrm{K}}=2.0 \mathrm{~s}\right)$ such that the zero-shear viscosity comes out equal to $6.6 \mathrm{MPa} \mathrm{s}$ (see above). Obviously, the Kuhn segment relaxation time so determined is also indispensable to compare model predictions to data in the nonlinear range. Table 1 reports both the discrete Fraenkel spectrum and the glassy one. The latter was obtained by fitting the glassy moduli (red curves in Figure 1) by again using Reptate.

Table 1. Discrete Spectra for PS27 at $115^{\circ} \mathrm{C}$

\begin{tabular}{ccccc}
\multicolumn{2}{c}{ Fraenkel chain $(N=30)$} & & \multicolumn{2}{c}{ glassy contribution } \\
\cline { 1 - 2 } relaxation times $(\mathrm{s})$ & moduli $(\mathrm{MPa})$ & & relaxation times $(\mathrm{s})$ & moduli $(\mathrm{MPa})$ \\
5.9 & 0.13 & & $3.9 \times 10^{-2}$ & $2.1 \times 10^{0}$ \\
1.5 & 0.21 & & $3.3 \times 10^{-3}$ & $7.3 \times 10^{1}$ \\
0.48 & 0.37 & & $5.6 \times 10^{-4}$ & $2.3 \times 10^{2}$ \\
0.20 & 0.41 & & $6.1 \times 10^{-5}$ & $1.7 \times 10^{2}$ \\
$7.2 \times 10^{-2}$ & 1.2 & & $6.4 \times 10^{-6}$ & $1.0 \times 10^{2}$ \\
$5.4 \times 10^{-3}$ & 0.60 & & $4.8 \times 10^{-7}$ & $7.8 \times 10^{1}$ \\
$2.6 \times 10^{-3}$ & 12 & $6.7 \times 10^{-8}$ & $2.0 \times 10^{1}$ \\
$1.2 \times 10^{-3}$ & 48 & & \\
\hline
\end{tabular}

The storage and loss moduli reported in Figure 1 (green curves) were finally obtained as a sum of the Fraenkel and glassy contributions. The green and black curves essentially coincide, as expected.

3.2. Startup of Uniaxial Elongational Flows. Figure 2 reports the startup elongational data of Matsumiya et al. ${ }^{6}$ for several values of the Hencky strain rate $\dot{\varepsilon}$ (from 0.01 to $0.5 \mathrm{~s}^{-1}$ ) and shows simulation results obtained both with and without monomeric friction reduction (full and dashed lines, respectively), the former with no time delay $\left(\zeta_{\text {eff }}=\zeta\right)$. The friction reduction parameters adopted in eq $6\left(S_{\mathrm{c}}=0.095, \alpha=\right.$ $2.3, n=2.4)$ were found by fitting the steady-state viscosity data by trial and error (see Figure 4 below). For all predictions in Figure 2, the glassy contribution has been added simply as a steady-state elongational viscosity, $\eta_{\mathrm{E}, \mathrm{G}}=3 \sum_{i} G_{i, \mathrm{G}} \tau_{i, \mathrm{G}}$, where the $G_{i, \mathrm{G}}$ 's are the glassy moduli reported in Table 1 , while the $\tau_{i, \mathrm{G}}$ 's are the glassy relaxation times of Table 1 reduced in proportion to the friction ratio as in eq 8 . Taking the glassy contribution at its steady-state value is justified by the fact that even the longest equilibrium glassy relaxation time is significantly smaller than $1 \mathrm{~s}$, the time where data start in 


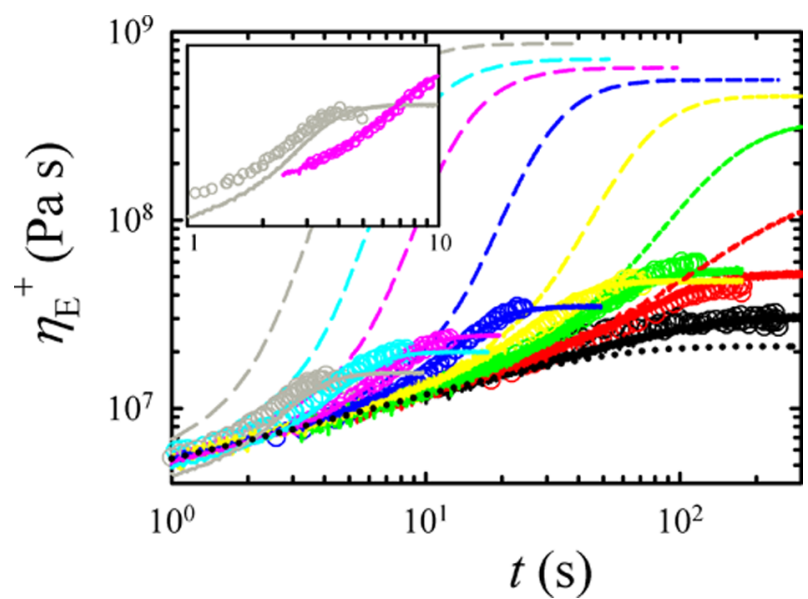

Figure 2. Transient elongational viscosity vs time during startup at several values of $\dot{\varepsilon}\left(0.01,0.02,0.03,0.05,0.1,0.2,0.3,0.5 \mathrm{~s}^{-1}\right.$, from right to left in different colors). Symbols are data from Matsumiya et al. ${ }^{6}$ Solid and dashed lines are model predictions with and without friction reduction, respectively. The dotted black line is the LVE prediction obtained using the spectra in Table 1. Model predictions do not account for time delay; hence, the inset highlights the significant discrepancies existing at short times for $\dot{\varepsilon}=0.5 \mathrm{~s}^{-1}$ (gray). Discrepancies already disappear for $\dot{\varepsilon}=0.2 \mathrm{~s}^{-1}$ (pink).

Figure 2. Consistently with previous works, ${ }^{3-6}$ comparison of dashed and solid lines in Figure 2 clearly shows the importance of monomeric friction coefficient reduction in fast elongational flows of PS melts. Indeed, model predictions without $\zeta$ reduction (dashed curves) run much higher than data.

Predictions accounting for $\zeta$ reduction (solid curves in Figure 2) are however not completely satisfactory. Indeed, during the transient, predictions run to the right of the data by a factor that increases significantly with increasing $\dot{\varepsilon}$ (see inset of Figure 2), suggesting the need of introducing a time delay in the monomeric friction reduction effect, as already noted by Matsumiya et al. ${ }^{6}$ Figure 3 then reports the predictions obtained using the full model, i.e., by also using eqs 7 and 8 , which contain the additional parameter $\tau_{\text {eq }}$ characterizing the time delay between $\zeta_{\text {eff }}$ and $\zeta(S)$ in transient flows. The curves reported in Figure 3 are obtained by choosing $\tau_{\text {eq }}=\tau_{\mathrm{K}}=2.0 \mathrm{~s}$,

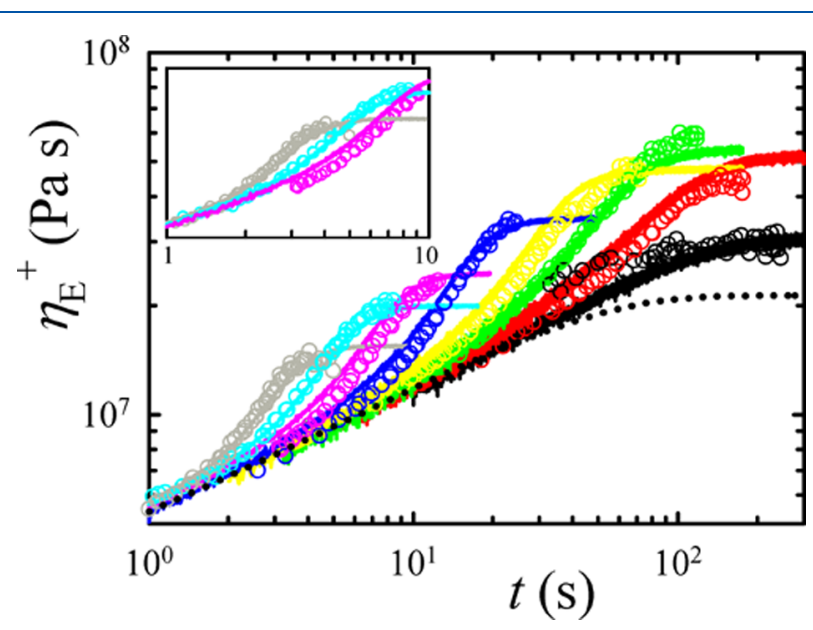

Figure 3. Transient elongational viscosity as in Figure 2. Here, however, solid lines are model predictions including time delay with $\tau_{\mathrm{eq}}=\tau_{\mathrm{K}}=2.0 \mathrm{~s}$. The inset better shows details for the three highest stretch rates. i.e., with the equilibrium delay time $\tau_{\text {eq }}$ equal to the Kuhn segment equilibrium relaxation time (previously reported). Predictions so obtained are generally in good agreement with data, also during the initial transient of the fastest flows (see inset of Figure 3), with the possible exception of $\dot{\varepsilon}=0.2 \mathrm{~s}^{-1}$ where predictions run slightly to the left of the data.

To better emphasize the key role of $\zeta$ reduction in fast uniaxial extensional flows of unentangled PS melts, Figure 4

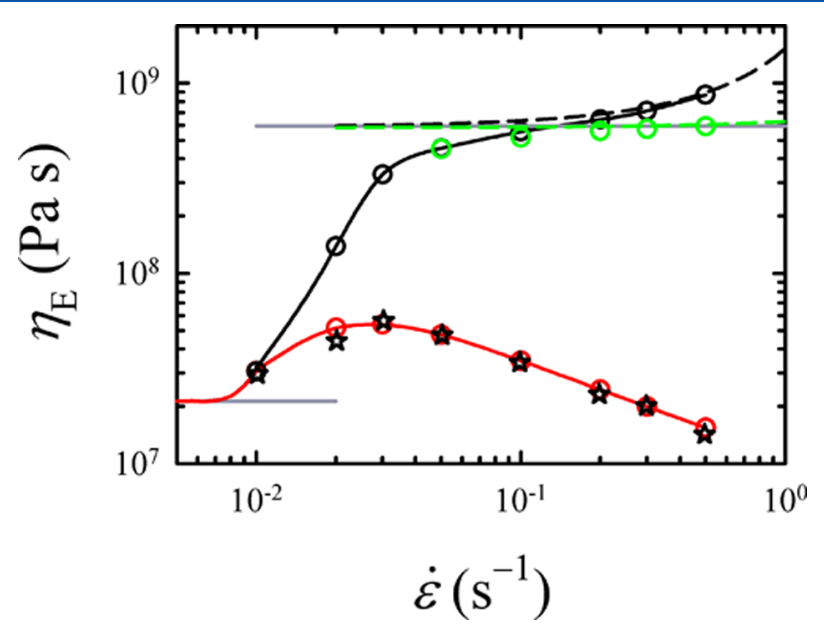

Figure 4. Steady-state elongational viscosity vs stretch rate. Black stars are data from Matsumiya et al. ${ }^{6}$ Red circles (and the red spline through them) are model predictions from Figure 3. Black circles (and the black spline through them) are model predictions without $\zeta$ reduction from Figure 2. Green circles are model predictions without $\zeta$ reduction but with $\tilde{H}=5000$ (rather than 500). Dashed black and green lines are viscosities of fully extended Fraenkel chains with $\tilde{H}=$ 500 and 5000, respectively. The lower gray line is the LVE limit, while the upper one gives the viscosity of fully aligned bead-rod chains.

compares model predictions with data for the steady-state elongational viscosity $\eta_{\mathrm{E}}$ as a function of the stretch rate $\dot{\varepsilon}$. Agreement between model predictions (red circles obtained from the long-time results in Figure 3) and data (stars) in Figure 4 is obvious since, as mentioned above, the $\zeta$-reduction parameters were determined by fitting predictions to the steady-state values of $\eta_{\mathrm{E}}$ data. However, Figure 4 clearly shows that predictions without $\zeta$ reduction (black circles obtained from the long-time results in Figure 2) run much higher than data (up to 2 orders of magnitudes). Black circles also reveal that the Fraenkel chain (with $\tilde{H}=500$ ) yields at the highest stretch rates, showing an upturn in the viscosity that runs even above the viscosity of the fully extended bead-rod chain (upper gray line in Figure 4). Yielding is however displaced to higher rates by increasing the spring constant to $\tilde{H}=5000$ (green circles in Figure 4). The yielding phenomenon of the Frankel chain is analyzed in the Appendix in the limiting case of fully extended chains, and results for $\tilde{H}=500$ and 5000 are reported as dashed lines in Figure 4. At low stretch rates, the dashed lines approach the upper gray line since Fraenkel chains are there equivalent to bead-rod chains. Conversely, at high stretch rates, Fraenkel chains approach the bead-rod limit only by progressively increasing the value of the spring constant.

The yielding phenomenon is clearly not observed when $\zeta$ reduction is accounted for because the tension in the chain is much reduced in such a case. Indeed, simulation results with $\zeta$ 
reduction in Figure 4 become $H$-independent already with $\tilde{H}=$ 500 (conveniently allowing for larger values of the time step).

3.3. Relaxation Following Startup of Elongational Flow. Figure 5 shows the comparison of model predictions

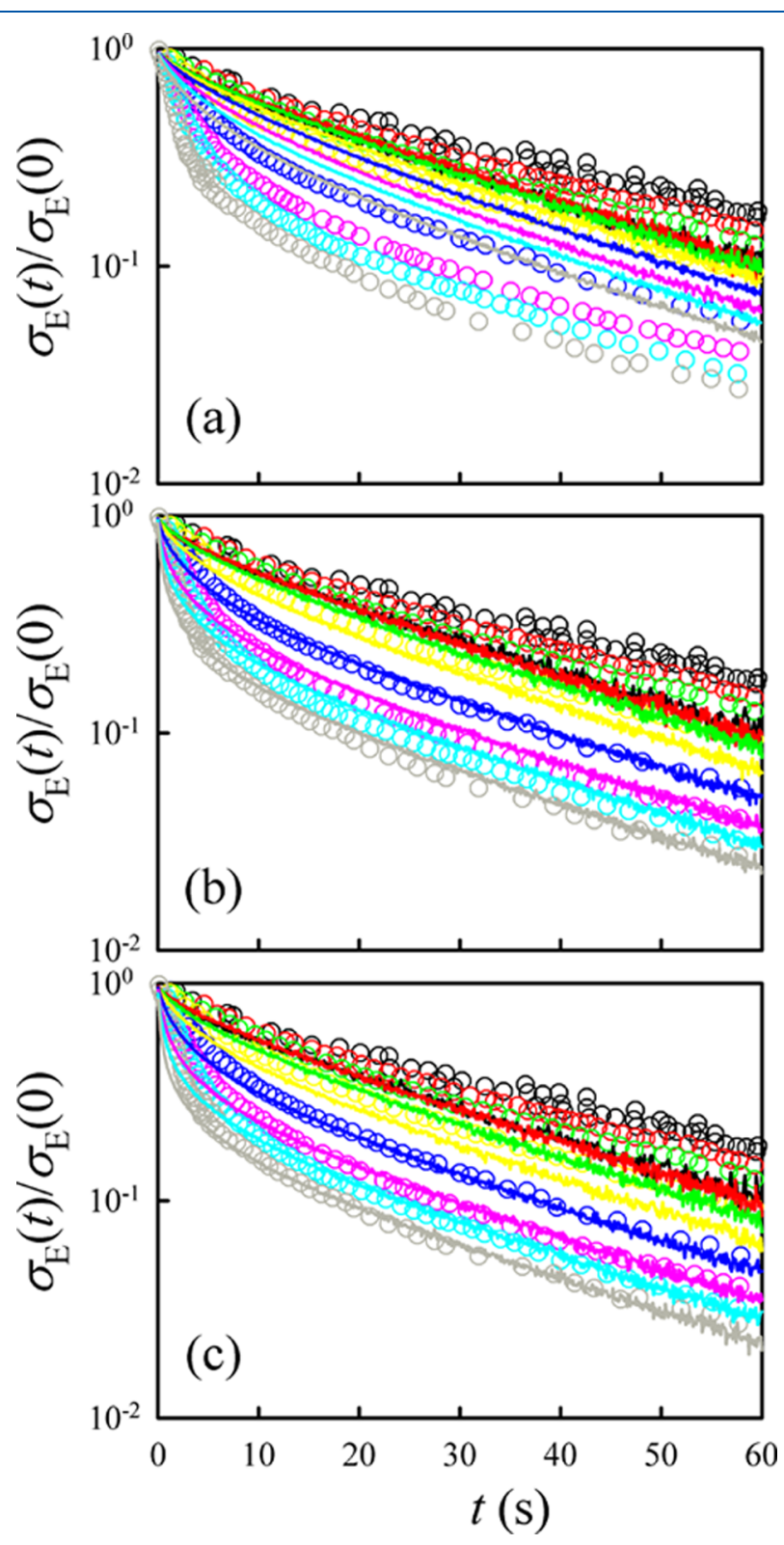

Figure 5. Stress relaxation after cessation of elongational flows up to $\varepsilon$ $=2.5$ at the same stretch rates as in Figures 2 and 3, increasing from top to bottom. Symbols are data from Matsumiya et al. ${ }^{6}$ Lines are model predictions (a) without friction reduction during relaxation, (b) with friction reduction but without time delay during relaxation, (c) with both friction reduction and time delay. The predicted stress $\sigma_{\mathrm{E}}(0)$ at the beginning of relaxation differs from that at the end of the startup by the glassy contribution (which relaxes virtually instantly).

with the relaxation data following elongational startup stopped at the Hencky strain $\varepsilon=2.5$ (for all values of $\dot{\varepsilon}$ as in Figures 2 and 3) from Matsumiya et al. ${ }^{6}$ Both data and predictions in Figure 5 are reported in terms of the ratio $\sigma_{\mathrm{E}}(t) / \sigma_{\mathrm{E}}(0)$, where $\sigma_{\mathrm{E}}(t)$ is the current value of stress and $\sigma_{\mathrm{E}}(0)$ is that at the beginning of relaxation (or nearly so for the case of data ${ }^{6}$ ). The latter differs from the stress at the end of the startup run by the glassy contribution, which relaxes in virtually no time.
Three different model predictions are considered in Figure 5. In Figure 5a, simulation results were obtained by restoring at the start of the relaxation the monomeric friction coefficient at its equilibrium value. Hence, as expected, predictions show a much slower relaxation than indicated by data. Again, $\zeta$ reduction proves to be indispensable.

Figure $5 b, c$ then reports predictions that include $\zeta$ reduction (with the same parameters of the startup) without time delay and including it, respectively. Here, agreement between predictions and data, though definitely better than in Figure $5 \mathrm{a}$, is only fair (and in fact similar in both figures). Indeed, at short times, predictions indicate a faster relaxation than shown by data (a feature to be further discussed below). At long times, although at high stretch rates prediction curves essentially run through the data, the latter indicate a somewhat slower ultimate relaxation. Indeed, the longest relaxation time of the model is the Rouse time, previously estimated at $33 \mathrm{~s}$ and essentially confirmed by the longest relaxation time in Table 1 (30 s). Conversely, the data in Figure 5 denounce a value of $43 \mathrm{~s}^{6}$ Such a difference between data and model predictions might either be due to the minor polydispersity of the PS sample (PI $=1.02)^{6}$ or to a minor influence of the topological interaction, not strong enough to be called entanglement, hence not visible in the shape of the frequency response in Figure 1. Indeed, the molar mass of PS27, though smaller than the PS critical molar mass $\left(M_{c} \approx 36 \mathrm{k}\right),{ }^{17}$ is significantly larger than the entanglement one $\left(M_{\mathrm{e}} \approx 18 \mathrm{k}\right),{ }^{6,13}$ thus making possible a minor topological effect.

Figure 6 reproduces Figure 5c with the time in log scale to better examine the behavior at short times. Figure 6 clearly

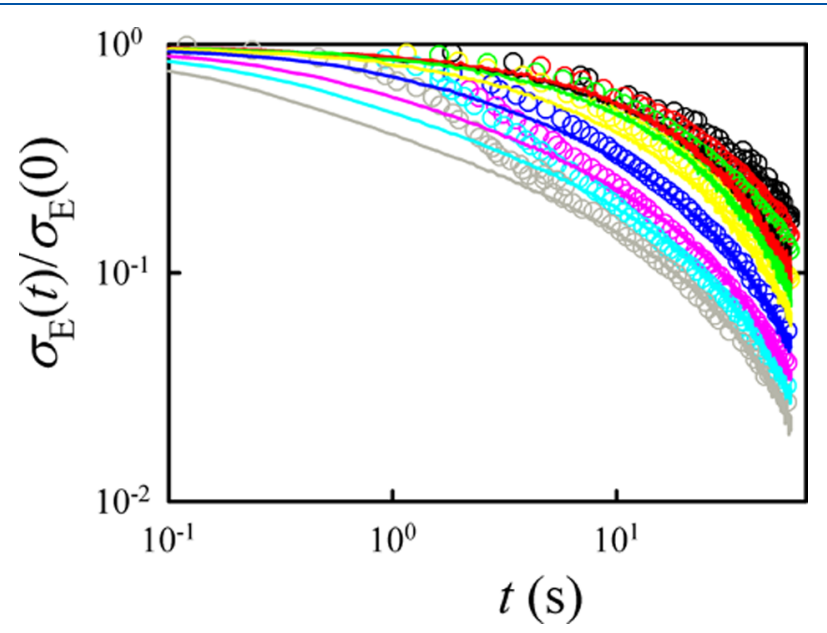

Figure 6. Same as Figure $5 \mathrm{c}$ with the time in log scale. Discrepancies between data and predictions are clearly visible at short times.

shows that, differently from predictions, the relaxation after flows at high stretch rates essentially takes place in two steps, the fastest of which occurs at a time on the order of the equilibrium Kuhn segment relaxation time, $\tau_{\mathrm{K}}=2.0 \mathrm{~s}$. Interpretation of such discrepancy between predictions and data does not seem easy. Excluding experimental artifacts, one possibility is to invoke a further role of Kuhn segment coalignment, namely, a change of the local interchain interactions (van der Waals attractions, etc.). On the other hand, it is known that local interchain interactions contribute to the stress tensor. ${ }^{18}$ To verify such an interpretation of the discrepancy in Figure 6, nonequilibrium molecular dynamics simulations 
would be required, which are beyond the scope of the present work.

The TOC figure, reporting the stress both in the startup at the highest stretch rate and in the subsequent relaxation (with the time in linear scale), also clearly reveals the discrepancy at the beginning of relaxation insofar as only the first $10 \mathrm{~s}$ of relaxation are reported. In the same TOC figure, the (small) sudden drop of the stress at the beginning of relaxation (green vertical segment at $t=5 \mathrm{~s}$ ) is due to loss of the glassy contribution occurring in a time interval too short to be observed.

\section{CONCLUSIONS}

The main conclusions of this work are as follows:

- The rheology of unentangled melts can be effectively simulated using Brownian dynamics of Fraenkel chains, similarly to the case of polymers in dilute solutions. Differently from solutions, however, a good quantitative fit to existing startup elongational data of unentangled melts can be obtained provided the monomeric friction coefficient is assumed to decrease with increasing the order parameter of the Kuhn segments of the polymer and with a delay time equal to the Kuhn segment relaxation time.

- The previous conclusion is in line with the existing results for entangled melts, where the flow-induced reduction of the monomeric friction coefficient is a welldocumented effect, but where however no time delay was observed. The reason for such a difference is apparent: indeed, relaxation times are much larger in entangled melts and hence time effects on the scale of the Kuhn segment are negligible in comparison and may well go unnoticed.

- The relaxation data after elongational startup of Matsumiya et al., ${ }^{6}$ when compared to the model predictions, reveal two discrepancies. One of them is the value of the ultimate relaxation time, which the data indicate somewhat larger than that assigned by the linear viscoelasticity results to either the Rouse chain or equivalently to the Fraenkel one. We suggest that such discrepancy might be explained by invoking a minor effect either of polydispersity or of weak topological interactions in the chain dynamics.

- The second important discrepancy is that between data and predictions at the beginning of the relaxation (see Figure 6). Data indicate that, after a fast elongational startup (strongly orienting the chains), essentially no relaxation takes place before times on the order of the equilibrium relaxation time of the Kuhn segment. A possible interpretation of this feature might invoke a flow-induced change in the local interchain interactions, similarly to the flow-induced change of the monomeric friction coefficient.

\section{APPENDIX}

Asymptotic Behavior of Bead-Rod and Fraenkel Chains We here report details on how we computed the viscosities (reported in Figure 4) of fully extended bead-rod and Fraenkel chains. In the fully extended limit, no ensemble average is required, and eq 3 gives the following expression for the elongational stress $\sigma$

$$
\sigma=\nu \sum_{i=1}^{N} F_{i} l_{i}=2 \nu \sum_{j=1}^{\bar{N}} F_{j} l_{j}
$$

where in the second equality symmetry has been invoked and we have renumbered chain segments starting from the center of the chain, with $\bar{N}=N / 2$ ( $N$ even is understood).

In the bead-rod case, segments have a fixed length equal to the Kuhn segment length $l_{\mathrm{K}}$, i.e., $l_{j}=l_{\mathrm{K}}, \forall j$. Tensions, on the contrary, are $j$-dependent, with a minimum at the chain end and a maximum at the center of the chain. The tension in the chain-end segments $(j=\bar{N})$ obeys the following force balance on the chain-end bead

$$
F_{\bar{N}}=\zeta_{\text {eq }} \dot{\varepsilon} \bar{N} l_{\mathrm{K}}
$$

where $\dot{\varepsilon} \bar{N} l_{\mathrm{K}}$ is the velocity of the medium surrounding the chain end with respect to the chain center (which is fixed). The force balance on the generic inner bead then reads

$$
F_{j}=F_{j+1}+\zeta_{\mathrm{eq}} \dot{\varepsilon} j l_{\mathrm{K}}
$$

so that the stress expression becomes

$$
\begin{aligned}
\sigma & =2 \nu l_{\mathrm{K}} \sum_{j=1}^{\bar{N}} F_{j}=2 \nu \zeta_{\mathrm{eq}} \dot{\varepsilon} l_{\mathrm{K}}^{2} \sum_{j=1}^{\bar{N}} j^{2} \\
& =2 \nu \zeta_{\mathrm{eq}} \dot{\varepsilon} l_{\mathrm{K}}^{2} \frac{\bar{N}(\bar{N}+1)(N+1)}{6}=\frac{\bar{N}(\bar{N}+1)(N+1)}{3} \\
& G \tau_{\mathrm{K}} \dot{\varepsilon}
\end{aligned}
$$

where, we recall, $G=v k_{\mathrm{B}} T$ and $\tau_{\mathrm{K}}=\zeta_{\mathrm{eq}} l_{\mathrm{K}}^{2} / k_{\mathrm{B}} T$. Since in the case of PS27, it is $N=30, G=0.12 \mathrm{MPa}$, and $\tau_{\mathrm{K}}=2 \mathrm{~s}$, we finally get $\eta_{\mathrm{E}}=6 \cdot 10^{2} \mathrm{MPa} s$ (see the upper gray line in Figure 4).

In the case of Fraenkel chains, chain segments have a uniform length, equal to $l_{\mathrm{K}}$, only in slow flows. With increasing values of the stretch rate, segment lengths become larger than $l_{\mathrm{K}}$, the less so, the larger the spring constant $H$. By setting $F_{\bar{N}+1}$ $=0$, eqs 11 and 12 then generalize to

$$
F_{j}=F_{j+1}+\zeta_{\mathrm{eq}} \dot{\varepsilon} \sum_{i=1}^{j} l_{i}, \quad j=1, \ldots, \bar{N}
$$

Since $F_{j}=H\left(l_{j}-l_{\mathrm{K}}\right)$, eq 14 can be written as

$$
l_{j+1}-l_{j}+\frac{\zeta_{\mathrm{eq}} \dot{\varepsilon}}{H} \sum_{i=1}^{j} l_{i}=0, \quad j=1, \ldots, \bar{N}
$$

where $l_{\bar{N}+1}=l_{\mathrm{K}}$ so that $F_{\bar{N}+1}=0$. Equation 15 defines a set of $\bar{N}$ linear equations in the $\bar{N}$ unknowns $l_{j}$ 's. The nondimensional parameter $\zeta_{\mathrm{eq}} \dot{\varepsilon} / H$ appearing in eq 14 can equivalently be written as $\dot{\varepsilon} \tau_{\mathrm{K}} / \tilde{H}$, where $\tilde{H}=H l_{\mathrm{K}}^{2} / k_{\mathrm{B}} T$ is the nondimensional spring constant. Finally, the stress is computed from eq 10 as

$$
\sigma=2 \nu H \sum_{j=1}^{\bar{N}}\left(l_{j}-l_{K}\right) l_{j}=2 G \tilde{H} \sum_{j=1}^{\bar{N}}\left(\tilde{l}_{j}-1\right) \tilde{l}_{j}
$$

with $\tilde{l}_{j}=l_{j} / l_{\mathrm{K}}$.

The $\dot{\varepsilon}$-dependent black and green dashed lines in Figure 4 were obtained from eqs 15 and 16 by setting $\tilde{H}=500$ and 
5000, respectively, and with the PS27 parameters $G=0.12$ $\mathrm{MPa}$ and $\tau_{\mathrm{K}}=2 \mathrm{~s}$.

\section{ASSOCIATED CONTENT}

\section{S Supporting Information}

The Supporting Information is available free of charge on the ACS Publications website at DOI: 10.1021/acs.macromol.9b00658.

Comparison of the friction reduction law proposed in the present paper to that used by Matsumiya et al. ${ }^{6}$ (PDF)

\section{AUTHOR INFORMATION}

\section{Corresponding Author}

*E-mail: iannirub@unina.it.

ORCID $\odot$

Giovanni Ianniruberto: 0000-0002-6963-8753

Antonio Brasiello: 0000-0002-3622-5258

Notes

The authors declare no competing financial interest.

\section{ACKNOWLEDGMENTS}

This work was partly supported by EU through the ITN DODYNET project (grant no. 765811). We also acknowledge kind assistance from the personnel of the SCOPE Computing Center of Federico II University.

\section{REFERENCES}

(1) de Gennes, P. G. Reptation of a Polymer Chain in the Presence of Fixed Obstacles. J. Chem. Phys. 1971, 55, 572-579.

(2) Doi, M.; Edwards, S. F. The Theory of Polymer Dynamics; Clarendon Press: Oxford, 1986.

(3) Ianniruberto, G.; Brasiello, A.; Marrucci, G. Friction Coefficient Does Not Stay Constant in Nonlinear Viscoelasticity. Proc. 7th Annual European Rheol. Conf. 2011, 1, 61.

(4) Ianniruberto, G.; Brasiello, A.; Marrucci, G. Simulations of Fast Shear Flows of PS Oligomers Confirm Monomeric Friction Reduction in Fast Elongational Flows of Monodisperse PS Melts As Indicated by Rheooptical Data. Macromolecules 2012, 45, 8058-8066.

(5) Yaoita, T.; Isaki, T.; Masubuchi, Y.; Watanabe, H.; Ianniruberto, G.; Marrucci, G. Primitive Chain Network Simulation of Elongational Flows of Entangled Linear Chains: Stretch/Orientation-Induced Reduction of Monomeric Friction. Macromolecules 2012, 45, 27732782.

(6) Matsumiya, Y.; Watanabe, H.; Masubuchi, Y.; Huang, Q.; Hassager, O. Nonlinear Elongational Rheology of Unentangled Polystyrene and Poly(p-Tert-Butylstyrene) Melts. Macromolecules 2018, 51, 9710-9729.

(7) Masubuchi, Y.; Yaoita, T.; Matsumiya, Y.; Watanabe, H.; Ianniruberto, G.; Marrucci, G. Stretch/Orientation Induced Acceleration in Stress Relaxation in Coarse-Grained Molecular Dynamics Simulations. Nihon Reoroji Gakkaishi 2013, 41, 35-37.

(8) Bird, R.; Curtiss, C.; Armstrong, R.; Hassager, O. Dynamics of Polymeric Fluids; Wiley: New York, 1987.

(9) Hsieh, C.; Jain, S.; Larson, R. Brownian Dynamics Simulations with Stiff Finitely Extensible Nonlinear Elastic-Fraenkel Springs as Approximations to Rods in Bead-Rod Models. J. Chem. Phys. 2006, 124, No. 044911.

(10) Ianniruberto, G. Extensional Flows of Solutions of Entangled Polymers Confirm Reduction of Friction Coefficient. Macromolecules 2015, 48, 6306-6312.

(11) Park, G. W.; Ianniruberto, G. Flow-Induced Nematic Interaction and Friction Reduction Successfully Describe PS Melt and Solution Data in Extension Startup and Relaxation. Macromolecules 2017, 50, 4787-4796.
(12) Inoue, T.; Okamoto, H.; Osaki, K. Birefringence of Amorphous Polymers. 1. Dynamic Measurement on Polystyrene. Macromolecules 1991, 24, 5670-5675.

(13) Rubinstein, M.; Colby, R. H. Polymer Physics; Oxford University Press: New York, 2003.

(14) Mark, J. E. Physical Properties of Polymers Handbook; AIP Press: Woodbury, 1996.

(15) Ramírez, J.; Sukumaran, S. K.; Vorselaars, B.; Likhtman, A. E. Efficient on the Fly Calculation of Time Correlation Functions in Computer Simulations. J. Chem. Phys. 2010, 133, No. 154103.

(16) Reptate, https://reptate.readthedocs.Io/.

(17) Léonardi, F.; Majeste, J.; Allal, A.; Marin, G. Rheological Models Based on the Double Reptation Mixing Rule: The Effects of a Polydisperse Environment. J. Rheol. 2000, 44, 675-692.

(18) Ramírez, J.; Sukumaran, S. K.; Likhtman, A. E. Significance of Cross Correlations in the Stress Relaxation of Polymer Melts. J. Chem. Phys. 2007, 126, No. 244904. 